\title{
Etude sur les lésions histo-pathologiques provoquées chez le cheval par l'anémie infectieuse.
}

\section{I. \\ Quelques observations sur le système nerveux. Cerveau et moelle épinière}

\author{
par \\ N. Nakamura, S. Ishil et S. Watanabe. \\ Institute vétérinaire du Gouvernement japonais, Tokio \\ (Cet ê sai est celui qui a rêproduit du rapport de l'Offce \\ International des Epizootiss en 1938.) \\ (:eçu pour la publication Sept. 1, 1938)
}

Intoroduction.-Basées sur la notion d'après laquelle le virus de l'anémie infectieuse du cheval a la propriété de stimuler les parois differenciées et non différenciées des vaisseaux sanguins, diverses études d'histologie-pathologique ont été entreprises en vue de rechercher principalement les lésions produites dans la moelle des os, le foie, la rate, les ganglions lymphatiques, les reins, les poumons, le coeur, etc. Nous n'avons trouvé, en ce qui concerne le système nerveux central des chevaux malades, d'autre observation histologique que dans le rapport succinct sur l'épendymite de l'animal malade par Holz. Nous avons souvent constaté, dans nos travaux expérimentaux relatifs à la recherche du virus dans l'organisme de divers chevaux, chez lesquels la maladie avait été provoquée par l'injection sous-cutanée de sang virulent, que la quantité de virus, suffisante pour faire apparaitre la maladie, n'était pas toujours présente dans le sang, le foie, la rate et les ganglions lymphatiques, mais qu'elle existait dans le cerveau, la moelle des os, aussi bien qu'en plusieurs organes endocriniens. Ces observations expérimentales nous ont incité à rechercher si des lésions histo-pathologiques pourraient être constatées dans le système ner- 
véux central. Notre travail est décrit ci-dessous.

\section{MÈTHODES EXPÉRIMENTAIES}

1. Animaux utilisés.-La maladie a été artificiellement provoquée chez 36 poneys de Corée, importés de l'île de Saishu-To, complètement indemnes d'anémie infectieuse. Six autres malades ont été choisis dans des régions infectées parmi des chevaux naturellement atteints. On a, en outre, utilisé les cadavres de trois sujets ayant succombé à des troubles de la nutrition ou à des maladies parasitaires. Enfin, six des chevaux consacrés, dans notre laboratoire, à la préparation de divers sérums immunisants, ont été ajoutés aux autres sujets de l'expérience pour servir de témoins.

2. Méthodes expérimentales.-La plupart des malades ont été abattus, mais quelques-uns d'entre eux moururent. Pour la fixation des fragments d'organes de plusieurs sujets, on a employé la solution de Zenker; la coloration était pratiquée par la méthode de Laidlaw. Mais, dans des buts divers, on a souvent recouru aux modes de fixation et de coloration suivants:

\begin{tabular}{|c|c|}
\hline Fixation & Coloration \\
\hline - & - \\
\hline Formaline..... & ... Hématoxyline-Eusine. \\
\hline Formaline... & ...Rouge écarlate. \\
\hline Alcool & Unna-Pappenheim. \\
\hline Regaud & Heidenhein-Fer-Hématoxyline. \\
\hline Zenker & Lentz. \\
\hline
\end{tabular}

Anatomie pathologique macroscopique.

Bien que des lésions macroscopiques du système nerveux central ne soient pas toujours nettement démontrées, on observe d'habitude de la congestion de la membrane méningée. Dans le cas où un ramollissement du tissu cérébral est constaté, les lésions histo-pathologiques dépendent $\mathrm{du}$ degré du ramollissement. On trouve rarement des taches hémorragiques, mais le liquide céphalorachidien est en général plus abondant. 


\section{Constatations histo-pathologiques.}

En ce qui concerne les lésion histo-pathologiques du système nerveux central, la maladie est vraisemblablement caractérisée par une inflammation non suppurative et par des processus de dégénérescence que l'on constate habituellement dans diverses maladies causées par les virus dits pseudo-neurotropes. Bien que les lésions dépendent de la durée de la maladie, on trouve, en règle générale, dans les prélèvements, une prolifération de cellules de la névroglie, de la neuronophagie, un afflux de leucocytes dans les vaisseaux, une dégénérescence des cellules nerveuses, de l'infiltration cellulaire périvasculaire, de la sub-épendymite et de l'épendymite, etc.

En général, de telles lésions histologiques se rencontrent rarement au stade de début de la maladie, tandis qu'il est démontré qu'elles se manifestent au fur et à mesure que la maladie progresse. Les descriptions succinctes données ci-dessous résument nos observations sur les lésions histologiques qui apparaissent aux divers stades de la maladie.

a) Prolifération des cellules de la névrnglie.-Une prolifération des cellules de la névroglie est généralement observée avant toute autre lésion histologique au stade de début de l'infection. La cellule névroglique que l'on rencontre à ce stade se différencie principalement en ce qu'elle est microglique diffusée dans toutes les parties du cerveau, mais surtout abondante sous le tissu de l'épendyme et de sa région voisine, affectant la forme d'un petit bâtonnet ou d'haltère, quelquefois celle d'un petit nodule.

Dans le matériel prélevé sur des malades présentant de fréquents accès fébriles, la prolifération diffuse de la cellule névroglique est particulière, car celle-ci prend même l'aspect des cellules macrogliques (principalement des cellules étoilées). Dans la couche superficielle du noyau Caudé (nucleus caudatus), la formation de ce qu'on appelle "étoiles névrogliques (astrocytes)" est démontrable. En outre, de telles trouvailles sont également faites dans le tissu du ventricule latéral, de l'aqueduc (de Sylvius) aussi bien que du bulbe olfactif, etc., tissu où l'on trouve de la sub-épendy- 
mite avec prolifération microglique. Quelquefois, sa légère infiltration sous la pie-mère, sa formation en petits nodules dans la zone moléculaire du cervelet et son infiltration périvasculaire dans la couche superficielle du nucleus caudatus peuvent être également démontrées.

Ainsi, la prolifération diffuse des cellules névrogliques dans le nucleus caudatus peut se montrer différente suivant l'état de l'animal au moment de l'examen.

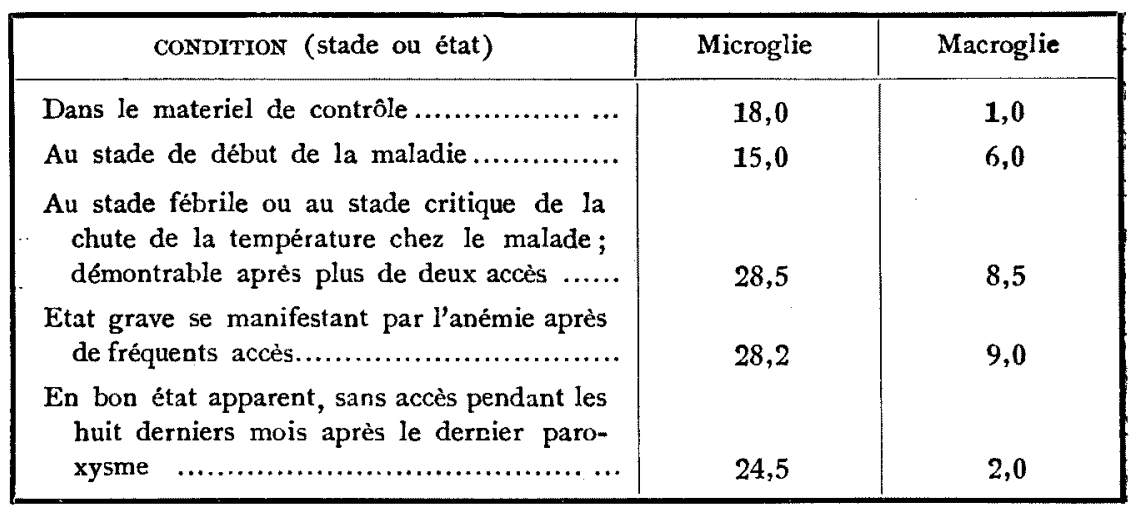

Les nombres ont été établis avec une moyenne de quatre champs optiques (champs microscopiques), l'épaisseur de la préparation étant de $5 \mu(1 \times 125)$.

Bien que le phénomène de neuronophagie soit également observé au cours de la maladie, il faut noter qu'il peut être mis en évidence, même chez le cheval qui, depuis longtemps, n'a pas eu d'atteinte fébrile. La neuronophagie se constate habituellement dans l'écorce cérébrale, spécialement dans le lobe frontal. Mais les mêmes modifications peuvent être observées dans une partie de l'hippocampe, dans la capsule interne, dans le noyau lenticulaire, dans les tubercules quadrijumeaux et dans la moelle allongée (bulbe rachidien), bien que, dans la règle, elles n'y soient pas aussi marquées (pseudoneuronophagie). L'attaque des cellules nerveuses par ces cellules névrogliques est d'ordinaire limitée aux petites ou -moyermes cellules, et elle porte très rarement sur les grandes cellules. 
Dans ces conditions, certaines cellulès nerveuses étant complètement dévorées ne peuvent se présenter que sous l'aspect d'un petit groupe de cellules microgliques.

b) Afflux des leucocytes dans les vaisseaux encépha'iques.-La mobilisation des leucocytes dans les vaisseaux sanguins du cerveau. est nettement démontrable dans l'anémie infectieuse, bien que le nombre de ces cellules varie avec la partie de l'organe examiné et avec le stade d'évolution de la maladie. On a observé qu'elle est le plus marquée dans la capsule interne du nucleus caudatus et dans les lobes frontaux de l'écorce cérébrale. Mais, elle est moins accentuée dans l'hippocampe, aussi bien que dans d'autres parties du cerveau. Ce processus est plus apparent dans la veine que dans l'artère, et il est démontré que les leucocytes sont plus nombreux dans les grosses veines. En conséquence, l'accumulation intense des leucocytes dans le veine peut être souvent rencontrée dans les cas graves.

L'afflux des leucocytes dans les vaisseaux est moins démontrable au stade de début de la maladie, mais, au cours d'un accès fébrile ou à la fin d'un tel accés, renouvelé plus de deux fois, on peut constater une augmentation du nombre de ces cellules. Néanmoins, si le malade s'affaiblit par suite d'une anémie grave, ou s'il passe huit mois sans attaque fébrile, on observe que le nombre des leucocytes diminue graduellement. En ce qui concerne liespèce des leucocytes observés, ce sont les histiocytes qui ont été habituellement décelés au stade de début de la maladie. Le nombre de ces cellules tend généralement à augmenter en récurrence de nouvel accès fébrile. Au contraire, à la fin d'une atteinte de la maladie, accompagnée d'anémie grave, ce sont les cellules lymphoides qui semblent être en augmentation. Au moment critique de la chute de la température, on trouve, d'ordinaire, des leucocytes éosinophiles.

c) Infiltration cellulaire perivasculaire.-L'infiltration cellulaire périvasculaire est une des modifications histo-pathologiques qui se rencontrent au cours de la maladie. Mais, comme on l'a observé, cette modification ne consiste généralement pas en une forte infiltration de cellules névrogliques. Naturellement, cettec onstatations 
n'est pas toujours la même dans tous les cas. Nous avons réparti en quatre groupes le degré d'infiltration observé chez 36 animaux examinés.

\begin{tabular}{lc}
\multicolumn{1}{c}{ Constatations. } & Nombre des cas. \\
Pas d'infiltration. & - \\
Légère infiltration. & 22 \\
Infiltration moyenne. & 7 \\
Infiltration marquée. & 4 \\
\end{tabular}

Par rapport à l'état du malade, l'infiltration peut être particulièrement bien observée dans les cas graves de la maladie, se manifestant par l'anémie, l'émaciation, ou un autre état encore plus grave, avec retour de l'accès fébrile, tandis que ce processus n'est pas observé dans les stades de début ou dans les atteintes modérées de la maladie. Quant aux points sur lesquels cette infiltration se produit, le premier envahi est en général la couche superficielle du nucléus caudatus; on ne la rencontre, du reste, nullement dans un autre tissu. Néanmoins, à mesure que les lésions progressent, une telle infiltration se répand dans une région étendue du cerveau. Dans ces cas, les modifications dans les diverses parties s'effectuent comme suit :

Comme on le voit au tableau qui suit, les modifications les plus importantes se trouvent, en général, dans tous les cas, dans le nucleus caudatus de même que dans l'hippocampe. De plus, il faut se souvenir que chez le $\mathrm{N}^{\circ} .129$, un des cas les plus graves de notre expérience, l'infiltration non seulement a pu être largement démontrée dans toutes les parties du cerveau, mais qu'elle a été aussi observée même dans les méninges de l'écorce cérébrale, de la moelle épiniére et du cervelet, où l'on constatait de la méningite. L'infiltration cellulaire des méninges a été observée chez les Nos 102 et 103 ; celle des parties lombaires spécialement a pu être nettement démontrée; elle s'étendait à toute la pie-mère depuis la portion du sillon ventral. En de pareils cas, on peut facilement supposer qu'un tel processus pathologique, atteignant les méninges et les cellules nerveuses de la région lombaire, est à même de provoquer la paralysie de l'arrière-train. 


\begin{tabular}{|c|c|c|c|c|c|}
\hline $\begin{array}{c}\text { CAS } \\
\text { oBservés }\end{array}$ & Nucl. Caud. & Hippocamp. & Rulb. Olfact. & $\begin{array}{c}\text { Tubercules } \\
\text { quadri. }\end{array}$ & $\begin{array}{c}\text { Trigo. } \\
\text { olfact. }\end{array}$ \\
\hline $\mathrm{N}^{\circ} .102$ & ++++ & ++-+++ & \pm & - & - \\
$\mathrm{N}^{\circ} .103$ & ++-+++ & ++ & \pm & \pm & ++ \\
$\mathrm{N}^{\circ} .129$ & ++++ & ++++ & ++ & +++ & +++ \\
\hline
\end{tabular}

\begin{tabular}{|c|c|c|c|c|c|c|}
\hline $\begin{array}{c}\text { CAS } \\
\text { ORSERvés }\end{array}$ & $\begin{array}{c}\text { Moelle } \\
\text { allongée }\end{array}$ & $\begin{array}{c}\text { Pedonc } \\
\text { céréb. }\end{array}$ & Punt & Cervelet & $\begin{array}{c}\text { Moelle } \\
\text { épin. }\end{array}$ & Mening. \\
\hline$N^{\circ} \cdot 102$ & - & & & - & & ++ \\
$N^{\circ} \cdot 103$ & \pm & ++ & + & - & - & + \\
$N^{\circ} \cdot 129$ & ++-+++ & +++ & ++ & ++ & +++ & ++++ \\
\hline
\end{tabular}

En ce qui concerne les cellules infiltrantes, on a observé que, dans le nucleus caudatus, au premier stade de la maladie, ce sont, d'ordinaire, les cellules de la microglie qui interviennent. L'infiltration périvasculaire est répartie dans l'espace lymphatique, qui entoure le vaisseau, sous forme d'anneau, ou en amas. On la rencontre quelquefois mélangée de cellules névrogliques en forme dite de baguette. Toutefois, à mesure que progresse le processus pathologique, les cellules infiltrantes sont rencontrées périvasculairement sur differents points. L'infiltration est surtout considérable dans la région des artères moyennes. De plus, elle se trouve en dehors des cellules de la tunique externe du vaisseau, non dans l'espace lymphatique, dans lequel on peut rencontrer, si l'on agit par pression, des cellules infiltrantes.

Par utilisation de la coloration Van Gieson, les cellules de l'adventice qui se multiplient et qui sont comprises dans le tissu conjonctif, peuvent être nettement mises en évidence. Les cellules observées ne sont pas des cellules microgliques, mais des cellules lymphoïdes avec un noyau radié; elles ressemblent aux petites cellules dites plasma-cells; leur teneur variable en protoplasma est manifests et la fragmentation de leur noyau est considérée comme un type de dégénérescence. Cependant, parmi elles, on ne rencontre guère les autres cellules qu'on trouve habituellement 
dans le sang.

d) Modifications dans les cellules nerveure.3. - Si des modifications des cellules nerveuses se rencontrent rarement à la période de début de la maladie, no observe, dans ces cellules, à mesure que cette maladie progresse, certains aspects pathologiques suivis processus inflammatoire habituel. Dans ces cas, les different de dégénérescence des cellules nerveuses peuvent être constatés; c'est quelquefois la "maladie aiguë des cellules", la "grave altération cellulaire de Nissl". Mais, en général, on ne rencontre pas ces modifications par groupes; elles sont le plus souvent éparses. On note avant tout, dans cette maladie, la dégénérescence des cellules nerveuses de la moelle épinière. D'après nos observations, ce processus peut être remarquablement démontré par la comparaison des petites cellules avec les grandes cellules nerveuses motrices. 11 semble que le processus de dégénérescence de ces cellules nerveuses puisse être suivi : premièrement, leur protoplasma et leur noyau et particulièrement ceux des grandes cellules, semblent atrophiés ; les cellules présentant une coloration foncée, et les corpuscules de Nissl, également de teinte foncée, en s'unissant les uns aux autres forment une "sorte de zone" (Zone like). Deuxièmement, le protoplasme et le noyau sont gonflés, distendus, et offrent un contour arrondi, ils semblent transparents, et les corpuscules de Nissl peuvent être vus sous une forme arrondie, présenter un aspect pâle, homogène, mais être considerablement diminues.

e) Hémorragie.-De même que les modifications pathologiques, l'hémorragie ne se rencontre pas dans tous les cas, mais on peut parfois rencontrer une hémorragie périvasculaire récente en forme d'anneau, dans diverses parties du cerveau ou près de l'aqueduc des tubercules quadrijumeaux, dans le nucleus caudatus, dans la couche superficielle de l'hippocampe, dans la corne latérale de la moelle épinière, etc. sans qu'il y ait, dans la règle, apparence d'infiltration cellulaire.

f) Inclusion de corps (enclaves).-1. Inclusion de corps dans les cellules de Purkinje.-Quelques corps se trouvent inclus dans le protoplasma ou dans le noyau des cellules de Purkinje. Ces corps sont de très petite dimension, d'environ $1 / 4$ ou $1 / 10$ du diamètre 
du nucléole; ils sont quelquefois entourés d'un halo non coloré. Dans la plupart des cas, on les rencontre disséminés dans une cellule, le plus souvent disposés à la périphérie, mais il est rare que leur nombre dépasse cinp, et le corps qui se trouve dans le nucleus est généralernent seul. Mais de tels corps peuvent être également démontrés dan la matériel prélevé sur des chevaux sains.

2. Inclusion de corps dans les cellules de l'épendyme--En ce qui concerne la forme et la dimension des corps inclus dans les cellules de l'épendyme, on ne trouve aucune différence entre eux et ceux qu'on observe dans les cellules de Purkinje; mais, dans quelques cas, par suite de leurs dimensions infimes, ils sont à peine visibles. On en rencontre, en général, un ou deux, parfois un groupe de dimensions diverses, de préférence dans le protoplasma des cellules qui avoisinent le canal central du tissu (canal de l'épendyme). D'habitude, la plupart d'entre eux se trouvent dans le quatrième ventricule; on les observe en nombre modéré dans le bulbe olfactif et le ventricule latéral, mais en très petite quantité dans la moelle épinière. Cette démonstration peut être faite d'une manière positive sur le matériel préléve sur l'animal malade, mais elle peut aussi être réalisée, bien que moins fréquemment, sur celui provenant d'un animal sain.

3. Inclusion de corps dans les cellules nerveuses.-L'inclusion de corps dans le protoplasme des cellules nerveuses peut être nettement mise en évidence dans le processus de dégénérescence du cerveau et de la moelle épinière. Les cellules nerveuses avec inclusion de corps sont principalement observées dans les différentes parties des centres, dont l'énumération suit :

Dans la partie antérieure du canal central du pont (de Varole), ou protubérance annulaire, autour de la pyramide bulbaire, dans la partie latéro-interne du canal central des tubercules quadrijumeaux, dans le pédoncule cérébral, le noyau caudé (nucleus caudatus) et la substance grise de la moelle épinière. On rencontre souvent aussi un petit nombre de tels corps dans quelques cellules nerveuses de l'écorce cérébrale, de l'hippocampe et du trigone olfactif. La forme de ces corps, de dimensions differentes, corres- 
pond à peu près à la description que nous avons donnée ci-dessus. Ces corps sont habituellement seuls ou par paires, mais quelquesuns sont aussi en groupes. Les cellules nerveuses, dans lesquelles les corps se trouvent groupés, se rencontrent parmi les petites cellules et elles sont caractérissés par des altérations dégénératives. D'autre part, les grandes cellules renferment d'ordinaire moins de petits corps, on en observe sans altérations. Dans les quelques endroits où les corps peuvent se trouver inclus dans le noyau de la cellule, pédoncule cérébral, moelle épinière, etc., ces corps sont généralement observés seuls, certains ressemblant, sous le rapport de la forme et de la dimension, aux corpuscules de Joest-Degen.

4. Inclusion de corps dans l'lippocampe.-D'après nos observations, les inclusions peuvent aussi, danscer tains cas d'anémie infectieuse, se rencontrer dans l'hippocampe. Elles existent habituellement dans quelques cellules nerveuses groupées en forme de fer à cheval. Fréquemment, leur dimension est celle du nucléole. Les cellules nerveuses, où il existe des inclusions, se trouvant généralement en voie de dégénérescence, le noyau de quelque-unes d'entre elles peut avoir disparu et les corps inclus, nombreux, peuvent être observés dans la cellule. Les corps que l'on rencontre dans ces cas sont d'ordinaire entourés d'un halo clair.

Il faut se rappeler, cependant, que la présence de semblables inclusions dans l'hippocampe peut aussi être relevée dans d'autres maladies.

\section{Discussion.}

Les observations histo-pathologiques faites dans differentes. parties du cerveau et du système nerveux des chevaux atteints d'anémie infectieuse montrent que le principal processus morbide paraît se dérouler dans les cellules nerveuses, le tissu mésodermique et les cellules de la névroglie de la partie antérieure du cerveau - noyau caudé (nuc'eus caudatus), capsule interne, noyau lenticulaire et lobes frontaux de l'écorce cérébrale, etc., - ces parties étant supposées subir l'action du virus. Les alterations histo-pathologiques principales sont la prolifération des cellules gli- 
ales ou névrogliques, l'afflux des leucocytes dans les vaisseaux, l'infiltration cellulaire périvasculaire, la neuronophagie, etc. Naturellement, en ce qui concerne l'apparition de ces lésions, des différences sont observées aux divers stades de la maladie. Par exemple, l'afflux des leucocytes dans les vaisseaux du cerveau se manifeste d'ordinaire nettement lors d'un accès fébrile ou après cet accès, et ce phénomène semble presque marcher de pair avec l'apparition d'histiocytes dans la circulation sanguine du malade. Les observations que nous avons faites au cours de nos expériences ne nous ont pas encore permis d'établir avec assez de certitude l'origine de ces histiocytes.

Se basant sur la description antérieurement donnée, Dunning et Furth (1935) ont écrit que les petites cellules de la microglie (microgliales) seraient identiques aux histiocytes au double point de vue morphologique et fonctionnel, et que les monocytes de la circulation sanguine ne peuvent être différenciés des deux formes de cellules précitées. En outre, Beizky a reconnu que la cellule histiocyte du mésenchyme de l'embryon de poulet est la même que les petites cellules de la microglie (cellules microgliques), que l'on rencontre dans les tissus nerveux, les histiocytes qui, par les vaisseaux, peuvent envahir la membrane chorioïdienne et la pie-mère, devenant ainsi des cellules microgliques. D'après nos observations, il est à noter que beaucoup de ces dernières cellules se trouvent dans la paroi des capillaires du tissu cérébral des animaux malades, tandis que les vaisseaux cérébraux de ceuxci contiennent de nombreux leucocytes qu'il est impossible de différencier des lymphocytes ou des histiocytes. L'apparition de telles cellules dans les vaisseaux du cerveau semble imputable à l'influence stimulante qu'exerce le virus sur la mobilisation cellulaire. L'infiltration cellulaire périvasculaire dans les diverses parties du cerveau doit être considérée comme le rèsultat de l'accumulation de cellules, due à la rèaction, directe ou indirecte, à l'action excitante $\mathrm{du}$ virus, les cellules infiltrantes provenant de la réaction directe, la première en date, étant les cellules de la névroglie, et celles issues de la réaction indirecte étant les cellules lymphoïdes qui tirent leur origine des cellules de l'adventice. 
De telles infiltrations sont habituellement observées en abondance dans la région des artères moyennes, où elles sont trouvées siégeant entre les cellules adventicielles. En ce qui concerne l'espèce des cellules, il n'est pas possible de mettre en évidence des leucocytes pourvus de granulations, ni aucune des variétés de leucocytes, que l'on trouve dans le sang. D'après nos observations, la méningite ne se rencontre guère au stade de début de la maladie. Mais, il faut considérer que la méningite peut apparaître lors d'une augmentation de la gravité du processus pathologique dans le tissu cérébral, comme nous l'avons constaté dans les cas 129 , 102 et 103 . Les diverses cbservations histo-pathologiques décrites ci-dessus peuvent généralement se rencontrer dans les différents cas où la maladie évolue sous la forme chronique, ou parfois lorsque le malade n'a présenté aucun symtôme clinique pendant plusieurs moị après le dernier accès ce fièvre.

Les lésions du tissu d'origine mésodermique peuvent être mises en évidence avant que n'apparaissent d'autres altérations sous la forme d'infiltration cellulaire de la paroi des vaisseaux, d'afflux leucocytaire dans les vaisseaux et d'augmentation des petites cellules de la microglie (cellules microgliques). On peut cependant relever un autre processus pathologique dans le tissu d'origine ectodermique, processus qui se traduit par une augmentation des grandes cellules de la névroglie (cellules macrogliques) et par la dégénérescence des cellules nerveuses. En accord avec de telles observations, on doit nettement reconnaître, comme l'a fait Dobberstein, que le virus de l'anémie infectieuse peut, au stade de début de l'infection, exercer une très grande action, son action principale, sur le tissu mésodermique et stimuler les parois non différenciées comme les parois différenciées des vaisseaux sanguins, et provoquer l'augmentation des histiocytes.

On peut ajouter à cette donnée que, au cours de l'évolution de la maladie, une altération peut également être observée dans le tissu ectodermique. L'inclusion de corps enclaves dans diverses cellules telles que les cellules de Purkinje, de l'épendyme et la cellule nerveuse ordinaire est nettement établie, mais nos connaissances actuelles ne nous permettent pas encore de déterminer la 
nature des corps en question. En ce qui concerne leur situation, ces enclaves se trouvent généralement dans le protoplasma de la cellule, mais quelquefois à l'intérieur du noyau. Dans la règle, les cellules avec corps inclus apparaissent sous une forme dégénérée, mais il faut observer que de tels corps peuvent aussi se rencontrer dans des cellules normales. N'oublions pas aussi que, d'après nos observations, de telles inclusions peuvent aussi être mises en évidence dans la cellule nerveuse du cheval, chez lequel l'inoculation artificielle du virus est restée sans résultat; toutefois, dans ce cas, les corps en question sont moins nombreux.

\section{Explication des clichés.}

Fig. 1. Neuronophagie dans les lobes frontaux de l'écorce cérébrale (cortex cerebri). Coloration de Laidlaw. Leitz. oc. 3 ob. $1 / 12$.

Fig. 2. Neuronophagie dans les lobes frontaux de l'écorce cérébrale (cortex cerebri). Coloration de Laidlaw, Leitz. oc, 3 ob, 1/12.

Fig. 3. Infiltration cellulaire périvasculaire de début dans la couche superficielle du noyau caudé (nucleus caudatus). Coloration de Laidlaw. Leitz. oc. 3 ob. $1 / 12$.

Fig. 4. Infiltration cellulaire périvasculaire dans le noyan caudé (nucleus caudatus) (au dernier stade). Culoration de Laidlaw. Leitz. oc. 4 ob. 4.

Fig. 5. Infiltration cellulaire dans la pie-mère de la partie lombaire de la moelle épinière. Coloration de Laidlaw. Leitz. oc. 4 ob. 4.

Fig. 6. Accumulation des leucocytes dans le vaisseau du noyau caudé (nucleus caudatus). Coloration de Laidlaw. Leitz. oc. 4. ob. 4.

Fig. 7. Inclusion de ccrps dans le protoplasma des tubercules quadrijumeaux (corpora quadrigemina). Coloration de Laidlaw. Leitz. oc. 3. ob. 1/12.

Fig. 8. Corps inclus dans le protoplasma d'une cellule de Purkinje. Culoration de Laidlaw. Leitz. oc, 3 ob. 1/12.

Fig. 9. Corps inclus dans le protoplasma de la cellule nerveuse de l'hippocampe. Coloration de Laidlaw. Leitz. oc. 3. ob. $1 / 12$.

Fig. 10. Corps inclus dans le noyau de la cellule nerveuse du noyau du pédoncule cérébral. Culoration de Laidlaw: Leitz. oc. 3. ob, 1/12.

\section{Bibliographie.}

(1) Holz: Perl. T. W.. 1937, 5; 65-68.

( 2) Dobberstein: Berl. T. W., 1934, 12, 192-196.

(3) Seifried: J. Exp. Med., 1931, 53, 2, 277-288.

(4) Larsell, Harling a. Meyer: Am. J. Path., 1934, 10, 3, 361-374.

(5) Dunning a. Furth: Am. J. Palh., 1935, 11, 6, 895-914.

(6) Dobberstein: Z. f. Jnf. para. Kh. u. Hyg. d. HI., 1928, 33, 290-305.

(7) Emoto, Kondo a, Watanabe : J. Jap. Suc. Vet. Sci., 1936, 15, 4, 41-62.

(8) Ishimitsu: Jap. J. Fxp. Med. 1937, 21, 8, 910-923.

(9) Ichii, Kato a. Wada: Juidampo., 1937, 339, 1221-1223. 


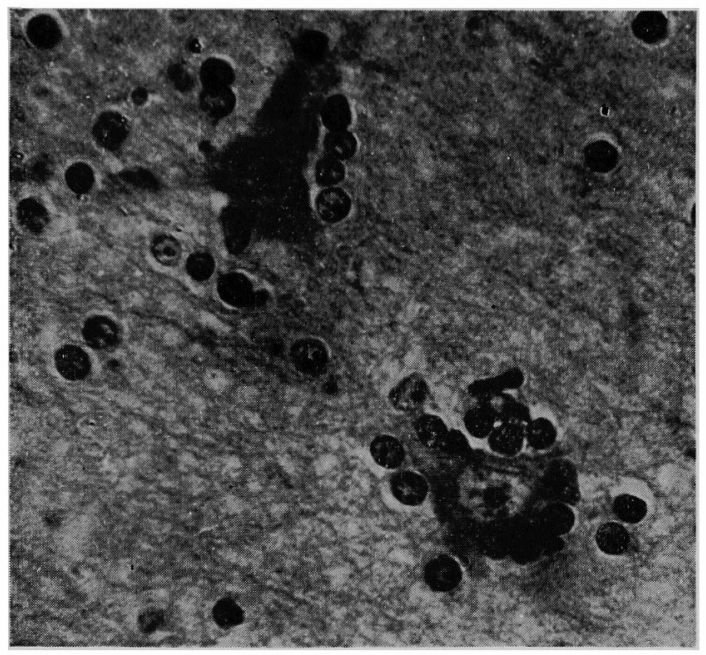

Fig. 1

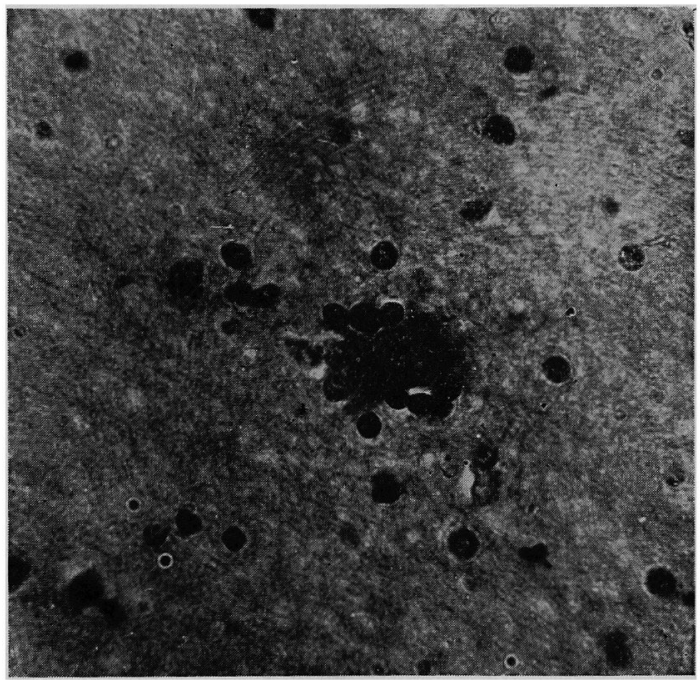

Fig. 2 


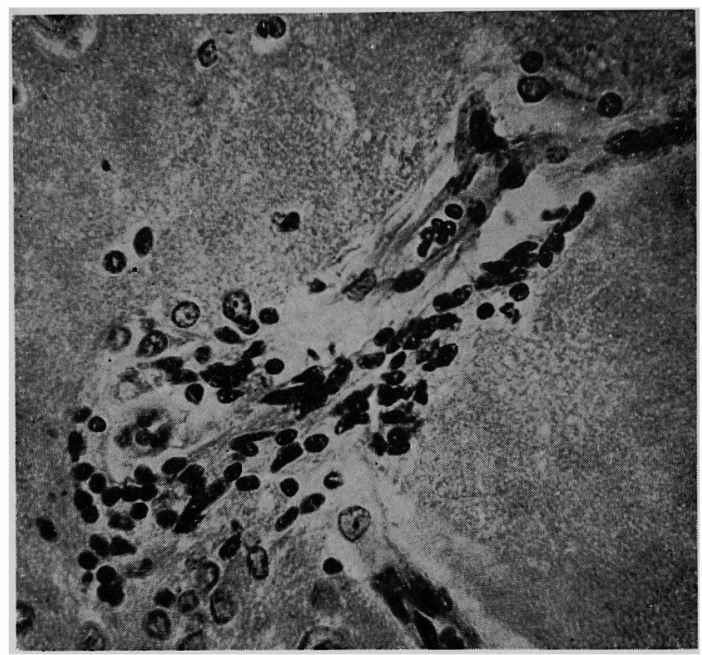

Fig. 3

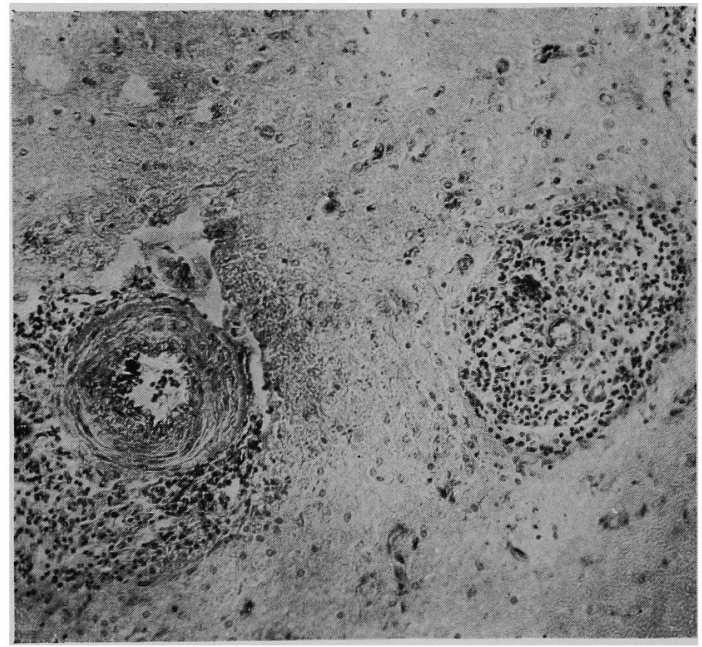

Fig. 4 


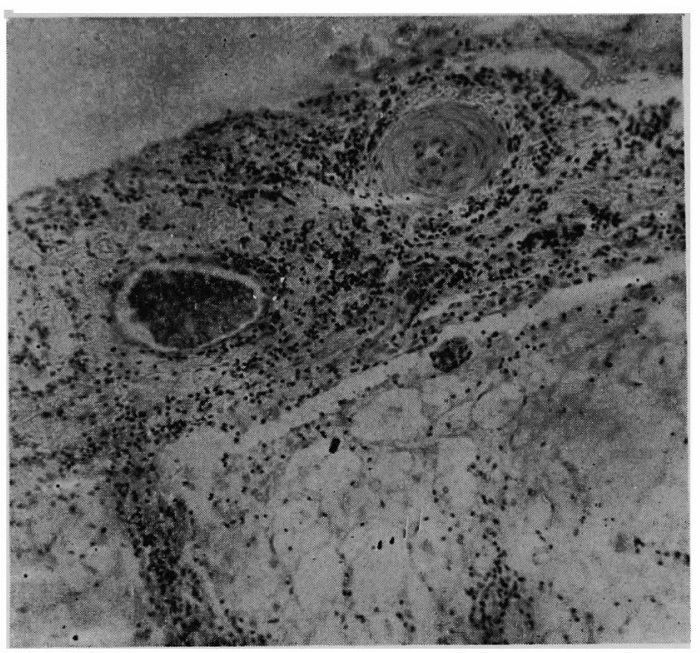

Fig. 5

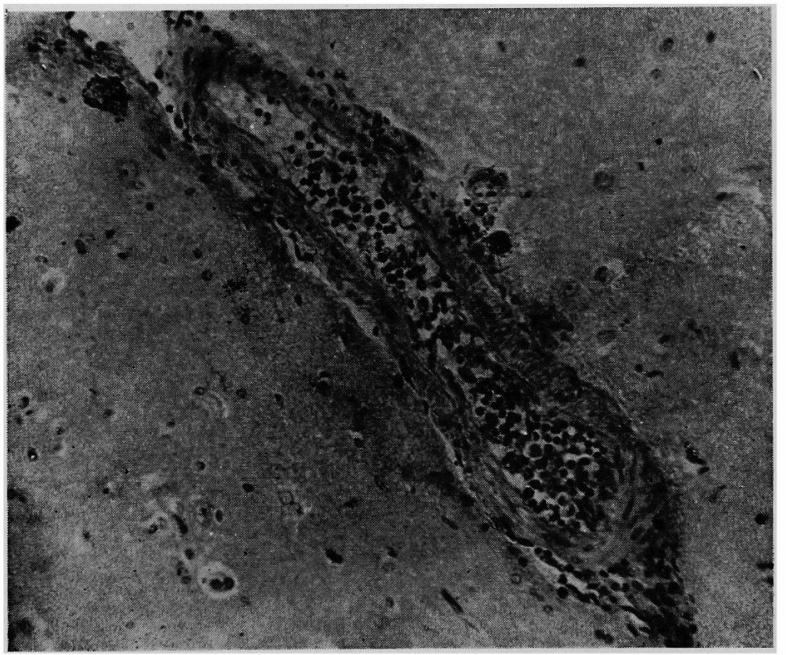

Fig. 6 


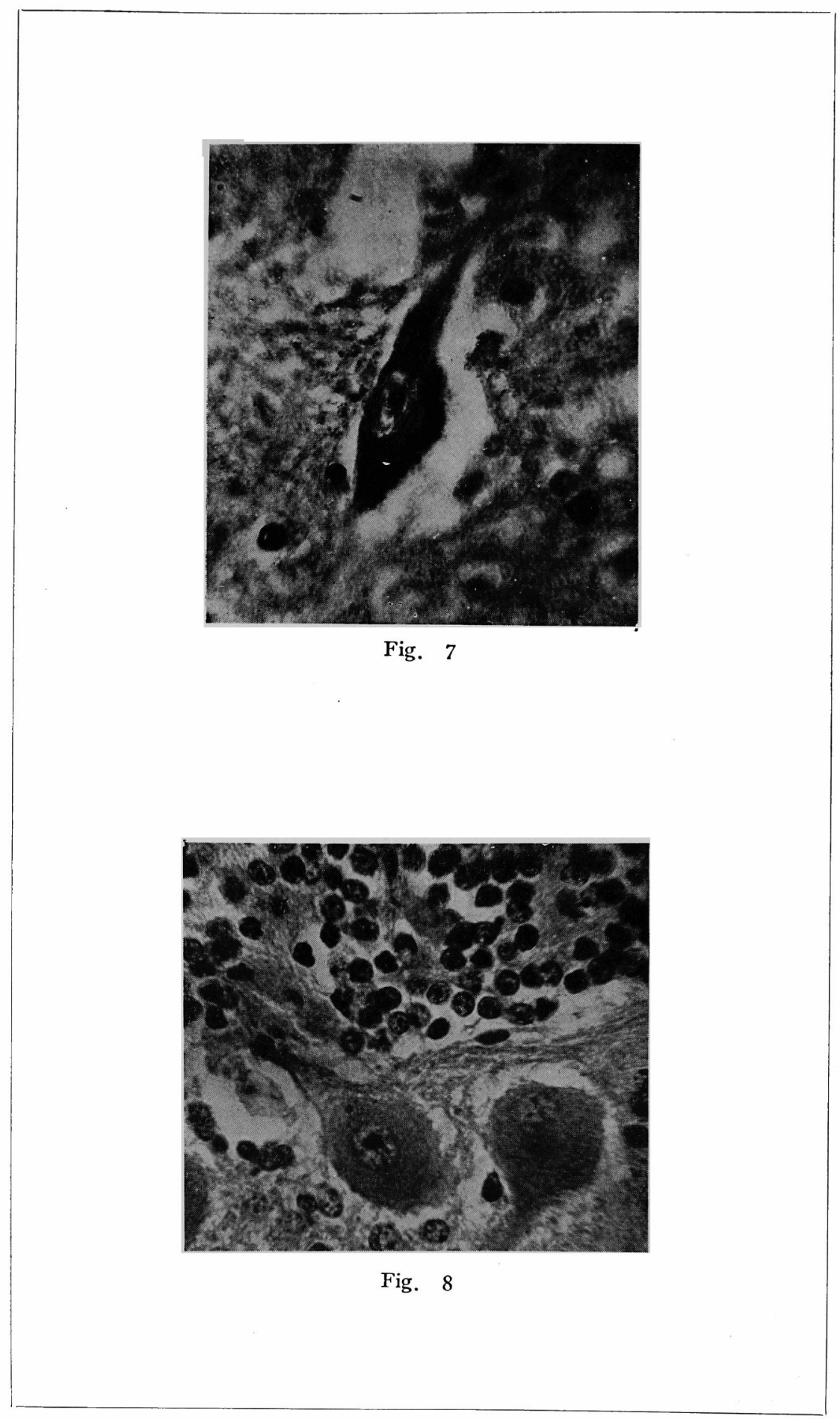




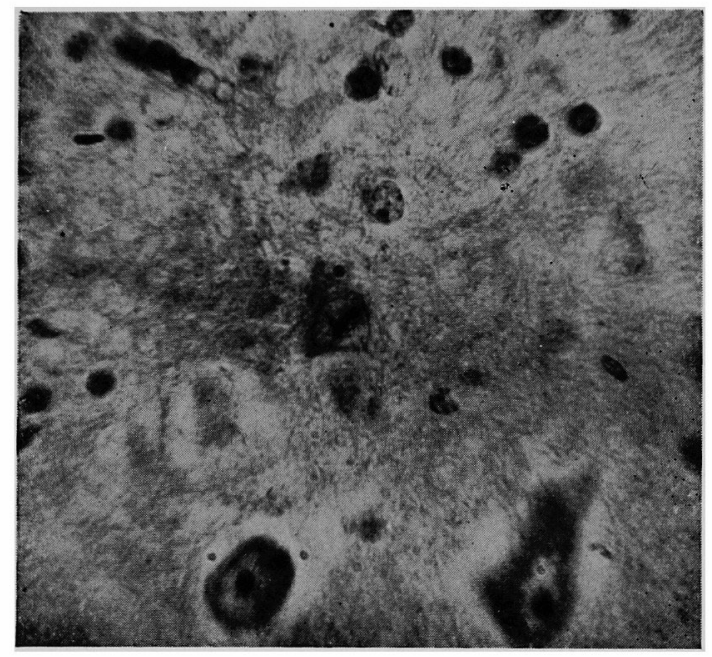

Fig. 9

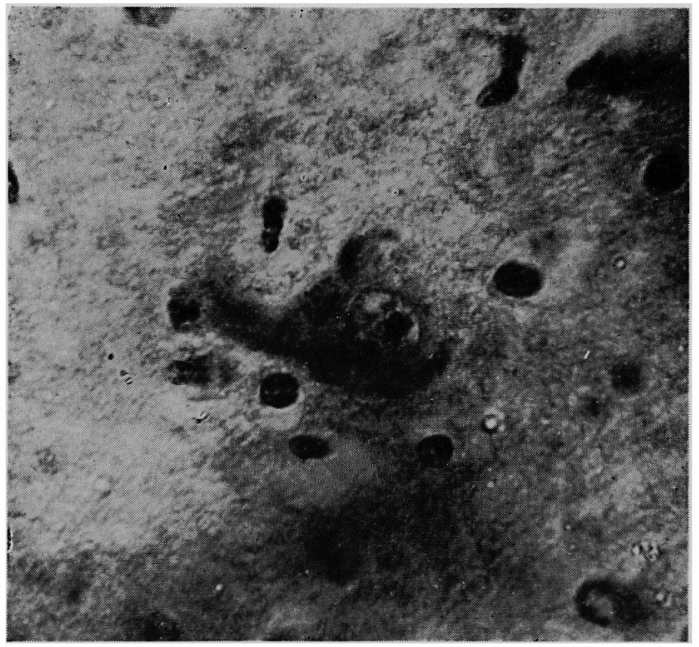

Fig. 10 


\section{傳染性貧血馬/病理組織學的研究*}

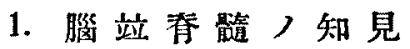

\section{中村哲哉石井進渡邊昇藏 \\ （農林省獸疫調查所）}

(昭和 13 年 9 月 1 日受附)

余等八別報本病經過馬二於ヶル病毒分布狀態 $=$ 關ス儿研究 $=$ 於テ記載シ タ如ク蓺發作ヨリ遠ザカツタ本病馬二於テ病毒が血液, 肝臓, 脾臓, 淋巴 腺等つ血液關係臟器 $=$ 證朋困難ナル場合却テ骨骨遀, 腦立立內分泌腺等二謴在 スル事實ヨ認メタノデアル右ノ如ク本病經過馬=於テ八病毒ガ永ク腦=存 在スル事實カラシテ之ニヨル病的變化ヨ豫想シ主トシテ本病清接種朝解馬

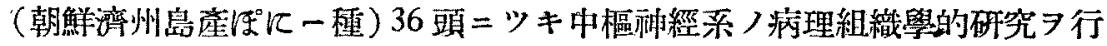
ツタ所甚ダ興味アル知見 7 得タノデソノ大要

郎于本病 $=$ 於テ所見スル中榀神經系統ノ組織學的變化八其ノ病期 $=ョ$ ツ テ異リ且ソノ程度入劣ルガ大體所謂或種ノ腦親和性病毒=認メ得ラレルヨ ウナ非化膿性炎症立退行性機轉ヨ知見シ得タノデアル

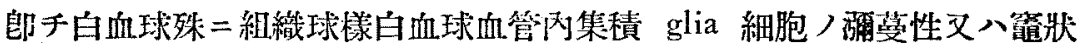
性墦殖立 neuronophagie 現象种經細胞ノ退行性變化血管周圍性細 胞集簇 等ノ變化ガ之デアル血管內=集積スル白血球八主トシテ組織球樣白血球デ 動脈ヨリ静脈二多クソノ程度入流血中ノ之等細胞卜渻長 $フ$ 同ジゥスル樣二 思ハレル

glia 細胞八游蒙性增殖幽 Pseudoneuronophagie 八比較的廣ク中樞䀡經系 統二認メラレタガ筑狀性增殖八主二尾狀核四疊體) cellule de l'épendyme つ附近又八小腦軟授膜下 = 買性 neuronophagie 八時二大腦皮質前頭部又八 れん柿核等二所見シタ

神經細胞ノ退行性變化八散發性二來ルコトガ普通デアルガ腰䯣, 奉髓=

*原著䟜文 64-76 頁學照 
於テハ集團的二來ルコトガ注目二價スル

血管周圍絧胞集族八比較的稀ニノミ所見セラレ動胍靜脈ヨ問ハズソノ周 園二認メラレルガ特=中等大動脈周園二多ク集皦細胞八車軸壯核 淋巴球樣細胞デ動脈二所見スルモノ八腫脹シタ血管外膜細胞間＝浸閵シテ ㄱ

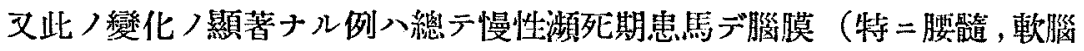
膜）二モ腦實質＝劣ラナイ細胞浸潤アリソノ程度ハソノ際認ム儿後體漹瘏 二比侧スルガ如キ結果

以上ノ諸所見八一般二病期つ初期二八認又難ク之ヨリ漸次經過スル ツテソノ程度ヨ垻シテクル之ヨ部位的二觀察スレバ尾狀核，あんもん核， 大腦皮質特二前頭部等ノ終腦部二著明デ他ノ部位=於テハ以上ノ變化八認 メラレルガソノ程度八比較的低イ

本患馬人中榀种經細胞就中あんもん核, えぺんでいむ細胞, ぷるきんえ 細胞立其入他各部神經細胞ノ原形質稀二細胞核內 =多クノ好酸性小體 見シタ

該小體ハ一般二多クノ本病䮈=所見シタガ非傳貧馬ニモ亦認メラレタノ デアル 